Correction

\title{
Correction: Wang, L., et al. Assessment of the Dual Polarimetric Sentinel-1A Data for Forest Fuel Moisture Content Estimation. Remote Sensing 2019, 11(13), 1568
}

\author{
Long Wang ${ }^{1}$, Xingwen Quan ${ }^{1, *} \mathbb{C}$, Binbin He ${ }^{1,2, *}$, Marta Yebra ${ }^{3,4}$, Minfeng Xing ${ }^{1} \mathbb{C}$ \\ and Xiangzhuo Liu ${ }^{1}$ \\ 1 School of Resources and Environment, University of Electronic Science and Technology of China, \\ Chengdu 611731, China; 201721180112@std.uestc.edu.cn (L.W.); xingminfeng@uestc.edu.cn (M.X.); \\ xiangzhuoliu@std.uestc.edu.cn (X.L.) \\ 2 Center for Information Geoscience, University of Electronic Science and Technology of China, \\ Chengdu 611731, China \\ 3 Fenner School of Environment and Society, The Australian National University, Canberra, ACT 2601, \\ Australia; marta.yebra@anu.edu.cn \\ 4 Bushfire \& Natural Hazards Cooperative Research Centre, Melbourne, VIC 3002, Australia \\ * Correspondence: xingwen.quan@uestc.edu.cn (X.Q.); binbinhe@uestc.edu.cn (B.H.)
}

Received: 19 December 2019; Accepted: 6 January 2020; Published: 7 January 2020

The authors wish to make the following corrections to this paper [1]:

1. Change in main body paragraphs

There is a mistake in this article. On page 6, lines 20-22 the sentence "Here, it should note that the $\sigma_{\text {soil }}^{o}$ in bare soil backscatter Linear model (Equation (6)) is expressed in linear unit while that in WCM (Equations (2) and (5)) is expressed in $\mathrm{dB}$ unit, and therefore the transformation between these two units was required." should be "Here, it should be noted that the $\sigma_{\text {soil }}^{o}$ in the bare soil backscatter linear model (Equation (6)) is expressed in $\mathrm{dB}$ units while that in the WCM (Equations (2) and (5)) is expressed in linear units, and therefore transformation between these two units was required.".

2. Change in figures

The authors wish to make the following corrections to this paper. Due to mislabeling, replace: 


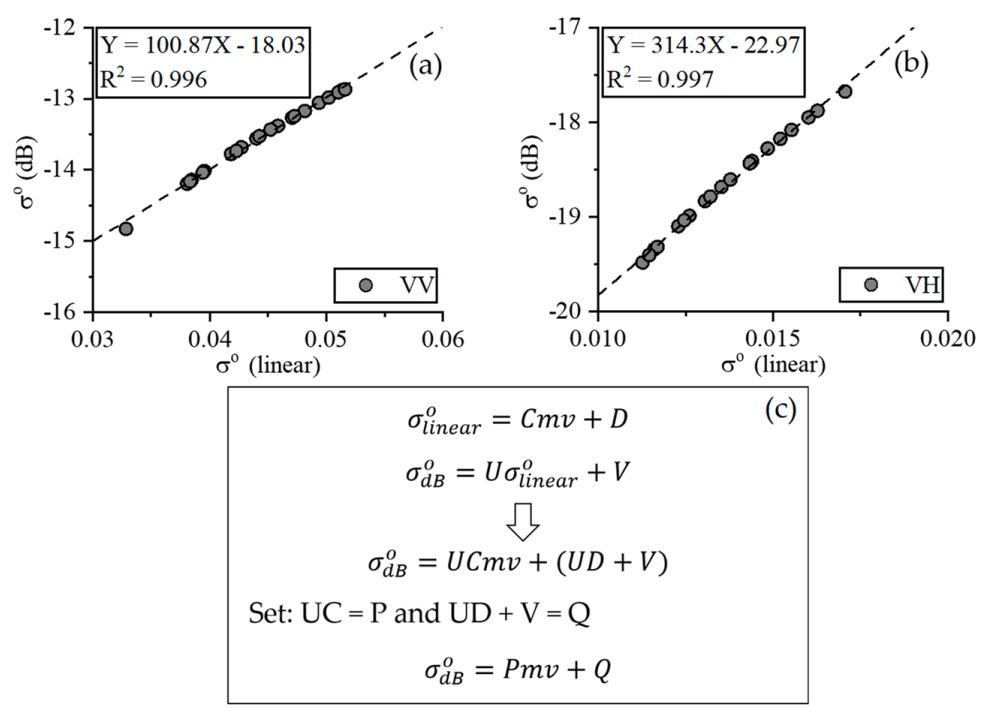

Figure 5. Linear relationship between backscatter in $\mathrm{dB}$ unit and that in linear unit over a small variation range for $\mathrm{VV}(\mathbf{a})$ and $\mathrm{VH}(\mathbf{b})$ polarization mode, and corresponding conversion formulas (c) used to reduce the model complexity.

With:

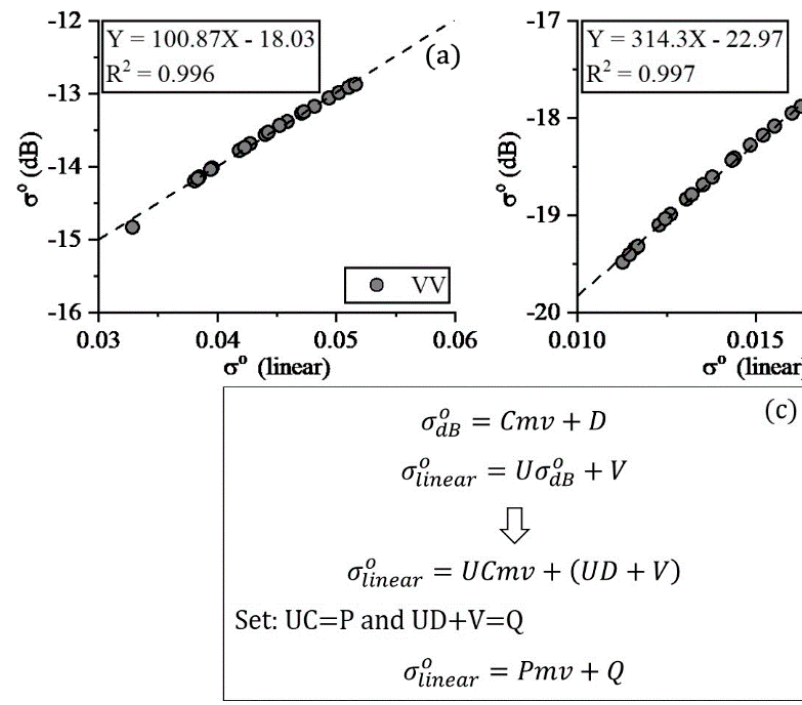

Figure 5. Linear relationship between backscatter in $\mathrm{dB}$ unit and that in linear unit over a small variation range for $\mathrm{VV}(\mathbf{a})$ and $\mathrm{VH}(\mathbf{b})$ polarization mode, and corresponding conversion formulas (c) used to reduce the model complexity.

The authors clarify that the above errors do not affect the formula of the final model (Equation (7)) and the experimental result of this article. The authors would like to apologize for any inconvenience caused to the readers by these changes.

\section{Reference}

1. Wang, L.; Quan, X.; He, B.; Yebra, M.; Xing, M.; Liu, X. Assessment of the Dual Polarimetric Sentinel-1A Data for Forest Fuel Moisture Content Estimation. Remote Sens. 2019, 11, 1568. [CrossRef]

(C) 2020 by the authors. Licensee MDPI, Basel, Switzerland. This article is an open access article distributed under the terms and conditions of the Creative Commons Attribution (CC BY) license (http://creativecommons.org/licenses/by/4.0/). 